\title{
Adaptive vector control based wave-to-wire model of wave energy converters
}

\author{
Antoni Cantarellas ${ }^{1,2} \bowtie$, Daniel Remon'2, Weiyi Zhang'2, Pedro Rodriguez ${ }^{1,2}$ \\ ${ }^{1}$ Abengoa Research, Abengoa, Campus Palmas Altas, Seville, Spain \\ ${ }^{2}$ Department of Electrical Engineering, Technical University of Catalonia, Gaia Building, Terrassa, Spain \\ 凶-mail: antoni.mir@abengoa.com
}

\begin{abstract}
This study presents a complete wave-to-wire model in which a novel wave energy converter control approach based on adaptive vector control is introduced. The proposed control for maximum power absorption of the primary resource is included, as well as the grid interconnection topology and required controllers, needed for processing the power over the entire wave energy conversion chain. Thanks to the adaptive performance of the proposed controller, maximum energy extraction can be instantaneously achieved regardless of the current irregular wave characteristics of the resource. Finally, the proposed electrical configuration arises as a suitable grid interconnection solution, as it not only provides maximum power supply from the wave energy resource, but it also contributes towards further reducing its output power oscillations.
\end{abstract}

\section{Introduction}

The development stage, in which the wave energy industry is found today, is characterised by the presence of a wide range of different wave energy extraction and conversion concepts. In this regard, the front conversion stages (primary energy capture and power-take-off (PTO) systems) have been the ones which comprise the major diversity of conversion designs, while the electrical conversion stage (electrical generator and power processing units) have been generically defined following a similar path than in the wind turbine industry [1-5]. Therefore, most of the research efforts have been focused on finding a widely-accepted high efficiency wave energy converter (WEC) concept with proven energy conversion technology, and sea performance capabilities under irregular sea states [6-9].

Considering the development stage of wave energy, in which commercialisation of several WEC concepts will become a reality in a close future, it is time for focusing part of the ongoing research on facing new technical and regulatory challenges regarding the control and grid interconnection of WECs. Therefore, the main challenges related to the control strategy applied to the WEC, and the design of power conditioning equipment required in order to provide acceptable power supply to the electrical grid should be successfully tackled [9].

Regarding the control of the wave energy resource, it is worth noting that multiple control solutions have been proposed in the literature with no clear sign of a straightforward preferred control candidate, resulting in maximum power extraction under realistic sea state conditions. Linear damping, latching and reactive based controllers $[6,10]$ initially appeared as suitable candidates in the frequency-domain control of regular waves, leading in the case of the reactive control to maximum energy absorption from the resource. However, this approach leads to sub-optimum conditions when applied to irregular realistic waves, as the system is tuned at the sea state level rather than at the wave-to-wave level. Other more advanced optimum and suboptimum time-domain based approaches appeared in [11-14] with the purpose of maximising the instantaneous energy absorption. However, such control strategies usually require an accurate characterisation of the incoming wave excitation force from far positioned measurement buoys, which are prone to introduce uncertainties or incorrect information depending on the distance to the WEC and on their interactions, if any.

Finally, one of the major concerns regarding grid integration of WECs is the limitation of the high power oscillations, experienced when performing maximum power extraction from the resource [15]. In addition to the undesired system integration performance, these power oscillations have a direct impact on the mechanical and electrical system overrating and its costs, as the energy conversion chain has to be designed to withstand high peak-toaverage power extraction rates. In order to limit such power fluctuations, many WECs include additional energy storage solutions in the mechanical/electrical PTO system, resulting in larger overall system costs.

Therefore, this paper contributes to the wave energy sector by proposing a novel WEC control concept, which is able to achieve maximum power absorption of the resource thanks to its inherent adaptive behaviour. The adaptive vector control approach arises as a suitable and robust solution, as it determines any control action, based on the self-velocity of the wave energy device and not based on the detailed knowledge from incoming waves. Thanks to the adaptive performance of the controller, the WEC is capable of achieving maximum power absorption regardless of the instantaneous performance of the resource, with no need of offline tuning parameters calculation depending on the incoming wave characteristics.

In addition, a suitable power processing and energy conversion chain has been proposed in order to provide an acceptable electrical grid interconnection system. Thanks to the smoothing effect from the DC interconnection of several WECs, the proposed system contributes towards reducing further the output power oscillations supplied to the grid, with no need of additional energy storage systems installed.

\section{Proposed wave-to-wire energy conversion structure}

This section introduces the proposed overall wave-to-wire energy conversion system in order to provide a general view of the particular scenario considered in this paper. The system configuration is depicted in Fig. 1.

The proposed WEC is built up around four heaving buoys, each of them connected to a hydraulic PTO. A cylindrical heaving buoy is used here as a generic WEC device, since the purpose of this paper is to introduce a novel WEC controller and its wave-to-wire system and not the study of the hydrodynamic efficiency of the device.

A hydraulic PTO system has been selected due to its inherent storage capacity, as it contributes to the overall capability for reducing the peak-to-average ratio. The main function of the 


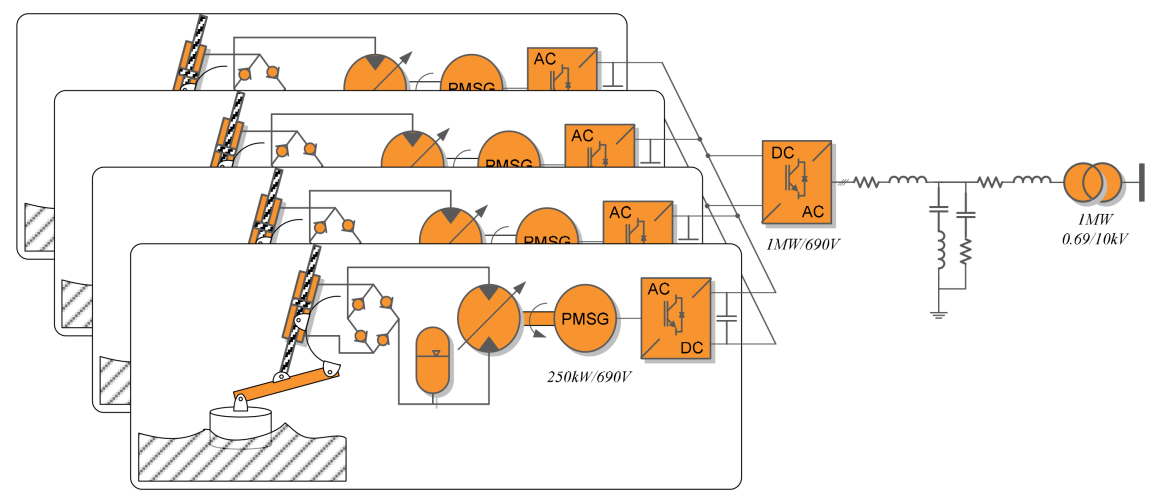

Fig. 1 Overall wave energy converter system configuration

accumulator is to smooth the flow rate fluctuations reaching the hydraulic motor, and thus reducing the peak-to-average ratio.

Each of the PTO systems drive a permanent magnet synchronous generator (PMSG), whose electrical power supplied to the grid is controlled through a back-to-back voltage source inverter (VSI). The selected technology ensures variable speed operation of the generator, while achieving successful grid voltage regulation and fault ride through performance $[9,16,17]$.

From Fig. 1, it is worth noting that the DC-link terminals of the PMSG drivers are interconnected together, and the overall WEC power is processed through a single front end VSI. By implementing this interconnection design it is possible to achieve an aggregated effect in the common DC link, since none of the power fluctuations of the heaving buoys will pulsate in phase with the others. Therefore, this feature allows for an extra reduction of the final peak-to-average ratio obtained in the electrical point of interconnection.

\section{Adaptive vector control of WECs}

\subsection{Characterisation of the irregular wave energy resource}

The real-time characterisation of realistic sea-performance is usually reproduced based on several sea-state parameters, which are provided as an input to the system. The output of the wave energy resource model is the excitation force, which will be considered as an input to the control system, and it is representative of the wave force applied to the buoy. The wave resource model is obtained in this case through the implementation of the Bretschneider wave energy spectrum. The sea-state parameters considered correspond to the ones from the EMEC facility, where the most probable significant wave heights $\left(H_{\text {wave }}\right)$ and dominant wave period $\left(T_{\text {wave }}\right)$ appear to be $1.47 \mathrm{~m}$ and $7.7 \mathrm{~s}$, respectively. Once the wave energy spectrum is obtained from (1)-(3) [18], the time-domain wave excitation force is calculated by using (1) and (2).

$$
\begin{gathered}
A=\frac{5}{16} \frac{H_{\text {wave }}^{2}}{T_{\text {wave }}^{4}} \\
B=\frac{5}{4} \frac{1}{T_{\text {wave }}^{4}} \\
S(f)=\frac{A}{f^{5}} \mathrm{e}^{-\left(B / f^{4}\right)} \\
\operatorname{Ampl}_{i}\left(\omega_{i}\right)=\sqrt{2 S\left(\omega_{i}\right) \Delta \omega} \\
F_{\text {exc }}(t)=\sum_{i=1}^{N} \operatorname{Ampl}_{i} \Re\left(\tilde{F}_{\text {exc }}\left(\omega_{i}\right) \mathrm{e}^{-\mathrm{j} \omega_{i} t+\mathrm{j} \varphi_{i}}\right)
\end{gathered}
$$

where $f$ are the spectrum frequency components considered $\tilde{F}_{\text {exc }}\left(\omega_{i}\right)$ are the frequency-domain radiation force coefficients obtained from a fluid dynamic software simulation; $\operatorname{Ampl}_{i}\left(\omega_{i}\right)$ are the frequency-domain excitation force amplitudes; $S\left(\omega_{i}\right)$ represents the magnitude of the wave energy spectrum for each frequency components, $\Delta \omega$ is the discretisation step of the wave frequency vector considered, and $\varphi_{i}$ represent the random phase angles associated to each frequency component of the vector.

\subsection{Hydrodynamic modelling of WECs}

The hydrodynamic interaction between the wave energy resource and the WEC can be modelled by representing all the forces affecting the system. In this regard, the time-domain equation of motion from (6)-(8) appears as a result of such interacting forces. The time-domain representation has been selected, as the frequency domain approach is no longer valid if real-time modelling and control of a WEC is pursued for realistic irregular sea applications $[19,20]$

$$
\begin{gathered}
{[M+A(\infty)] \ddot{x}(t)+\int_{0}^{t} K(t-\tau) \dot{x}(\tau) \mathrm{d} \tau+K_{S} x(t)} \\
=f_{\mathrm{exc}}(t)+f_{\mathrm{PTO}}(t) \\
K(t)=\frac{2}{\pi} \int_{0}^{\infty} B(\omega) \cos (\omega t) \mathrm{d} \omega \\
A=\lim _{\omega \rightarrow \infty} A(\omega)=A(\infty)
\end{gathered}
$$

where $[M+A(\infty)]$ represent the acceleration coefficients due to the mass of the device, the convolution term $\int_{0}^{t} K(t-\tau) \dot{x}(\tau) \mathrm{d} \tau$ represents the radiation force damping coefficient, and $K_{S} x(t)$ stands for the buoyancy coefficient of the device.

Since the WEC system will be modelled and controlled in real time, Matlab Simulink will be used. Then, it is necessary to develop the WEC control and model in the Laplace domain. From (6), it can be observed that the radiation force convolution $\left(\int_{0}^{t} K(t-\tau) \dot{x}(\tau) \mathrm{d} \tau\right)$ is the term introducing a higher complexity when determining the time-domain equation in Laplace. Hence, the radiation force transfer function introduced in (9) has been derived from the Prony approximation method applied to the radiation impulse response function of (7) $[15,20]$. Once this transfer function is characterised, the Laplace equation of motion can be introduced as in (10).

Where $[M+A(\infty)] \ddot{X}(s)$ represents the acceleration force due to the mass of the device, $H_{\mathrm{rad}}(s) \dot{X}(s)$ is the radiation force, $K_{s} X(s)$ is the buoyancy force, $H_{\mathrm{PTO}}(s) \dot{X}(s)$ is the PTO force exerted to the WEC, and $F_{\text {exc }}(s)$ is the force from the incoming waves. As it can be observed from (10), the WEC performance can be modelled by a mass-damper-spring model, where the power extracted from the system depends on the applied PTO force.

$$
H_{\mathrm{rad}}(s)=\frac{\sum_{i=0}^{8} \alpha_{i} s^{i}}{\sum_{i=0}^{8} \beta_{i} s^{i}}
$$




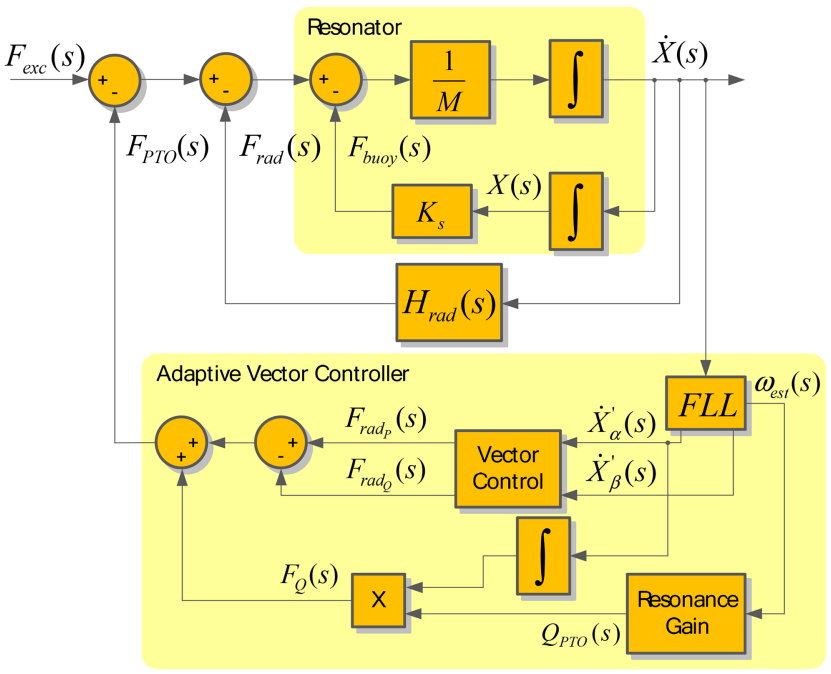

Fig. 2 Proposed adaptive vector controller of wave energy converters for maximum power absorption

$$
\begin{array}{llll}
\alpha_{8}=6704 & \alpha_{7}=2.812 \times 10^{5} & \beta_{8}=1 & \beta_{7}=1.922 \\
\alpha_{6}=6.352 \times 10^{5} & \alpha_{5}=4.675 \times 10^{6} & \beta_{6}=17.79 & \beta_{5}=26.66 \\
\alpha_{4}=7.163 \times 10^{6} & \alpha_{3}=1.777 \times 10^{7} & \beta_{4}=80.87 & \beta_{3}=73.39 \\
\alpha_{2}=1.393 \times 10^{7} & \alpha_{1}=1.119 \times 10^{7} & \beta_{2}=96.25 & \beta_{1}=38.35 \\
\alpha_{0}=5.613 \times 10^{5} & & \beta_{0}=20.12 &
\end{array}
$$

$$
\begin{aligned}
& {[M+A(\infty)] \ddot{X}(s)+H_{\mathrm{rad}}(s) \dot{X}(s)+K_{s} X(s)} \\
& +H_{\mathrm{PTO}}(s) \dot{X}(s)=F_{\mathrm{exc}}(s)
\end{aligned}
$$

\subsection{Proposed adaptive vector control approach}

According to [21], the optimum energy capture from the resource is achieved when the PTO force applied to the WEC cancels the inherent mass $(M+A(\infty))$ and buoyancy $\left(K_{s}\right)$ terms of the wave energy device. Under this operating condition, the system appears at resonance, which is a necessary condition for maximum energy absorption from the resource. In addition to resonance, the PTO transfer function should provide a resistive damping term equal to the real component of the radiation force transfer function $\left(\Re\left(H_{\mathrm{rad}}(s)\right)\right)$, otherwise optimal power extraction of the resource cannot be achieved.

The reactive or complex conjugate control arose as the control approach capable of ensuring such WEC performance. Therefore, the PTO provides a force with real part equal to the radiation force damping component, where the imaginary part ensures resonant conditions for the incoming wave frequencies. The major problem found from most controller implementations in the literature, is that its PTO terms are usually calculated offline, and according to a dominant frequency characteristic of the sea state, which is usually obtained from the excitation force characterisation of measuring buoys. This performance leads to suboptimal operation, as the system is tuned for a dominant wave frequency component, and not for the instantaneous wave-to-wave frequency spectra. In addition, the controller is very sensitive to the accuracy of the incoming wave characterisation, since any estimation error has a direct impact on the undesired controller performance $[10,14,15]$.

The proposed controller tackles all the aforementioned problems, as thanks to the adaptive frequency estimator used, the controller is capable of providing the required real-time PTO parameters for ensuring maximum energy absorption in a wave-towave basis, regardless of the incoming wave's characterisation. In addition, it arises as a robust solution, since the controller feedback is based on the instantaneous velocity of the device itself, and not on estimations from far reached measurement buoys [22]. Therefore, the adaptive vector controller proposed is introduced in Fig. 2 and is described in detail throughout this section.
The first representative contribution of the proposed controller is the implementation of a signal monitoring and synchronisation system, e.g. a frequency locked loop (FLL) system for the case under study. Such monitoring and synchronisation system cannot be considered a novelty itself, as it has been already implemented in many grid connected converter applications [23, 24]. However, the contributions are found in the implementation of this signal synchronisation system in the wave energy field, as it provides an instantaneous adaptive characterisation of the wave energy resource based on the movement of the WEC itself.

3.3.1 Signal monitoring and synchronisation system (FLL): In this particular case, the FLL structure is the responsible of instantaneously estimating the dominant frequency components $\left(\omega_{\text {est }}\right)$ of the WEC velocity, which are the frequency components to which the entire wave energy conversion system should resonate with in order to ensure optimal operation conditions. In addition, the FLL structure also has the capability of providing the direct and quadrature components of the buoy velocity vector $\left(\dot{x}_{\alpha}^{\prime}, \dot{x}_{\beta}^{\prime}\right)$, which will be used later in the proposed vectorial approach for determining the required PTO force.

As specified in $[23,24]$, the tuning parameters of the FLL are the SOGI gain $\left(K_{\text {SOGI }}\right)$ and the FLL gain $(\gamma)$, which determine the selectivity of the bandpass filter and its frequency tracking dynamics. In this specific case $K_{\text {SOGI }}=\sqrt{2}$ and $\gamma=0.16$ have been considered as a trade-off for ensuring high frequency detection dynamics, while providing accurate direct and quadrature components for the bandpass wave periods between 9.1 and $6.3 \mathrm{~s}$, respectively, which are used as the boundary wave periods for testing the suitability of the controller under different resource conditions.

3.3.2 Vector control of WECs: Once the direct and quadrature components of the buoy velocity $\left(\dot{x}_{\alpha}^{\prime}, \dot{x}_{\beta}^{\prime}\right)$ are determined, the active and the reactive power producing terms of the radiation force $\left(f_{\operatorname{rad}_{P}}, f_{\text {rad }_{Q}}\right)$ can be obtained by considering the following vectorial approach introduced in Fig. 3. The vector control approach proposed in this paper is also found as a novel contribution in the wave energy field. This novel approach aims at characterising the radiation force components which appear in phase $\left(\vec{f}_{\operatorname{rad}_{P}}\right)$ and quadrature $\left(\vec{f}_{\operatorname{rad}_{Q}}\right)$ with the buoy velocity vector $\left(\overrightarrow{\dot{x}}_{\text {wec }}\right)$, in order to instantaneously provide a resistive PTO force equal to the radiation force damping, while cancelling any possible reactive term introduced by the radiation transfer function $\left(f_{\text {pto }}=\left(\Re\left(H_{\text {rad }}(s)\right)-\mathfrak{\Im}\left(H_{\text {rad }}(s)\right)\right) \cdot \overrightarrow{\dot{x}}_{\text {wec }}\right)$. When applying such vectorial approach, the condition early mentioned of providing a resistive force equal to the radiation damping component is satisfied.

The first step for determining these active and reactive radiation force components $\left(\vec{f}_{\operatorname{rad}_{P}}, \vec{f}_{\operatorname{rad}_{Q}}\right)$ is the characterisation of the radiation force vector $\left(\vec{f}_{\text {rad }}\right)$ from its calculated orthogonal real and imaginary components $\left(\vec{f}_{\operatorname{rad}_{\alpha}}\right)$ and $\left(\vec{f}_{\operatorname{rad}_{\beta}}\right)$ from (11) and (12). As it can be observed in Fig. 3, the radiation force active powerproducing term $\left(\vec{f}_{\operatorname{rad}_{p}}\right)$ results from the radiation force projection over the buoy velocity vector (i.e. the radiation force component in-phase with the velocity vector), while the reactive powerproducing term $\left(\vec{f}_{\mathrm{rad}_{Q}}\right)$ appears as the radiation force projection inquadrature with it. Such radiation active and reactive power components can be determined according to (13) and (14).

The main advantage of the proposed vectorial approach is that, when obtaining the active and reactive radiation force terms, many power strategies could be used. For the case introduced in (13) and (14) maximum instantaneous power absorption will be achieved. However, if the interest is found in maximising the average power extraction, (15) and (14) apply. 


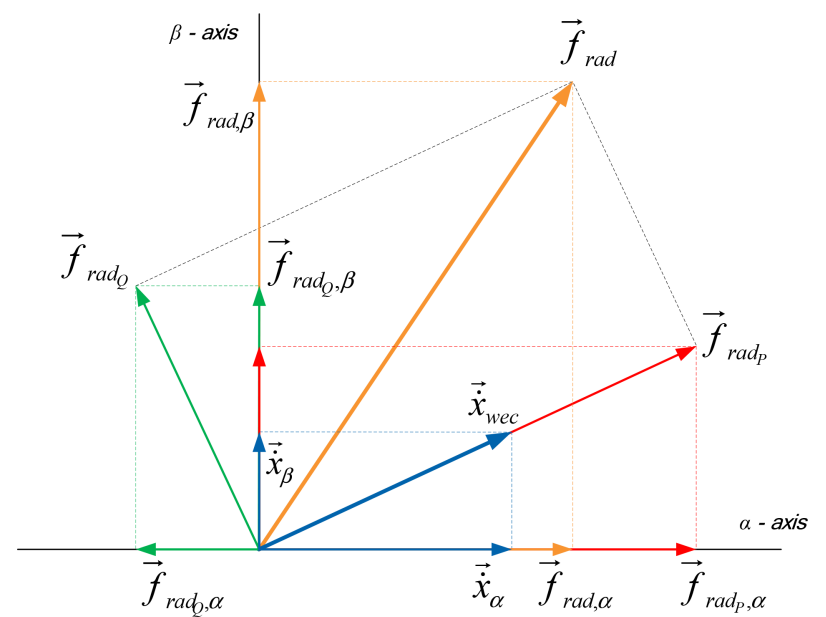

Fig. 3 Proposed adaptive vector controller of wave energy converters for maximum power absorption

$$
\begin{gathered}
\vec{f}_{\mathrm{rad}_{\alpha}}=H_{\mathrm{rad}}(s) \overrightarrow{\dot{x}}_{\alpha}^{\prime} \\
\vec{f}_{\mathrm{rad}_{\beta}}=H_{\mathrm{rad}}(s) \overrightarrow{\dot{x}}_{\beta}^{\prime} \\
\vec{f}_{\mathrm{rad}_{p}}=\frac{\overrightarrow{\dot{x}}_{\alpha}^{\prime} \cdot \vec{f}_{\mathrm{rad}_{\alpha}}+\overrightarrow{\dot{x}}_{\beta}^{\prime} \cdot \vec{f}_{\mathrm{rad}_{\beta}} \cdot \overrightarrow{\dot{x}}_{\alpha}^{\prime}+\dot{x}_{\beta}^{\prime 2}}{\vec{f}_{\mathrm{rad}_{Q}}=\vec{f}_{\mathrm{rad}^{\prime}}-\vec{f}_{\mathrm{rad}_{P}}} \\
\vec{f}_{\operatorname{rad}_{P}}=\frac{(1 / T) \int_{t}^{t+T}\left(\overrightarrow{\dot{x}}_{\alpha}^{\prime} \cdot \vec{f}_{\mathrm{rad}_{\alpha}}+\overrightarrow{\dot{x}}_{\beta}^{\prime} \cdot \vec{f}_{\mathrm{rad}_{\beta} \beta}\right)}{(1 / T) \int_{t}^{t+T}\left(\dot{x}_{\alpha}^{\prime 2}+\dot{x}_{\beta}^{\prime 2}\right)} \cdot \overrightarrow{\dot{x}}_{\alpha}^{\prime}
\end{gathered}
$$

3.3.3 Virtual PTO buoyancy term for resonance operating conditions: Finally, a virtual spring term should be provided by the PTO in order to ensure instantaneous resonance conditions regardless of the dominant frequency of the buoy velocity. From Fig. 4, a resonator structure can be observed between the intrinsic added mass and buoyancy terms of the wave energy device. The transfer function resulting from this resonator structure is

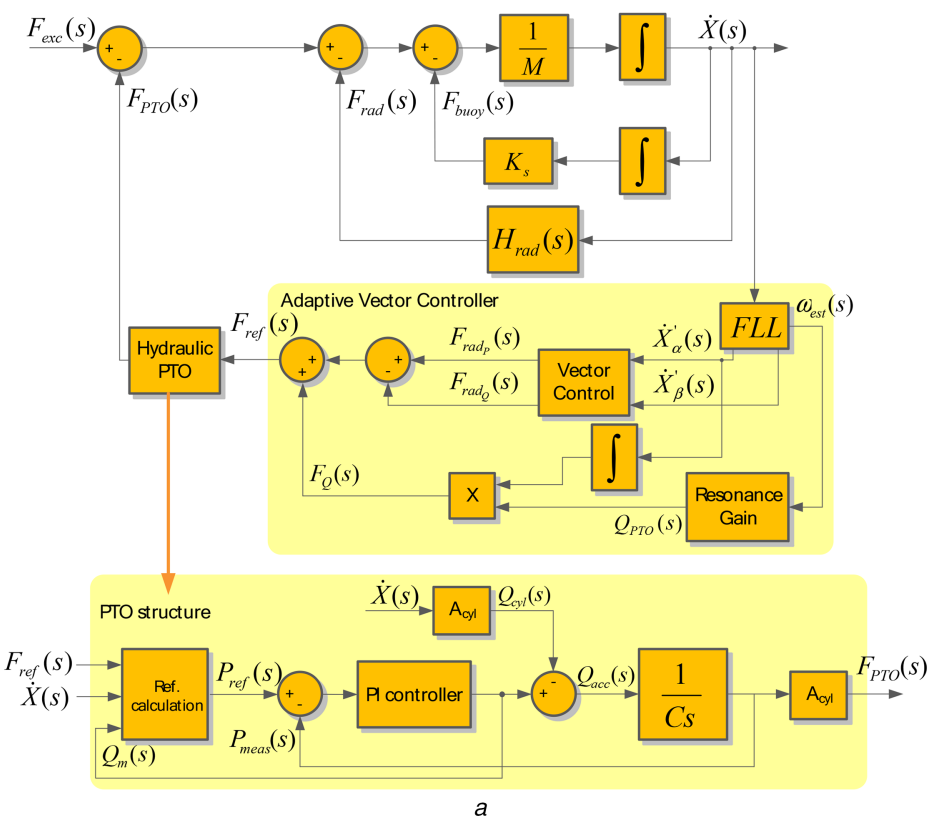

introduced in (16), from where the instantaneous calculation of the PTO spring coefficient $\left(Q_{\text {PTO }}\right)$ is given in (17). This spring PTO coefficient ensures instantaneous adaptive resonance conditions, since the frequency of the buoy velocity is instantaneously estimated from the FLL structure $\left(\omega=\omega_{\text {est }}\right)$.

$$
\begin{aligned}
H_{\text {res }}(s) & =\frac{s}{M s^{2}+K_{s}+Q_{\mathrm{PTO}}} \\
& =\frac{s / M}{s^{2}+\left(K_{s}+Q_{\mathrm{PTO}}\right) / M} \\
s^{2}+\omega^{2} & =s^{2}+\frac{K_{s}+Q_{\mathrm{PTO}}}{M} \\
& \Rightarrow \omega^{2}=\frac{K_{s}+Q_{\mathrm{PTO}}}{M} \\
& \Rightarrow Q_{\text {РTO }}=M \omega^{2}-K_{s}
\end{aligned}
$$

where $M$ represents the buoy mass term, $\omega$ is the estimated frequency from the FLL, and $K_{s}$ is the spring coefficient related to the buoyancy force of the WEC.

Therefore, maximum power absorption is obtained by implementing the proposed controller, as the PTO force is able to provide a real component equal to the real term of the radiation force, while the virtual spring term instantaneously cancels any possible interaction among the imaginary components, ensuring then resonance operation. This controller provides an adaptive solution thanks to the adaptive performance of the FLL system. The proposed WEC controller appears thus as a novel contribution in the wave energy field, since it provides an adaptive performance based on a vectorial approach for obtaining maximum power absorption from realistic ocean waves.

\section{Hydraulic PTO system modelling and control}

The hydraulic system considered in this section is introduced in Fig. 1, from which the hydraulic motor pressure is controlled by regulating the flow rate over the motor. In order to achieve the desired system performance, the PTO model and control introduced in Fig. 4 makes use of the reference force from the WEC controller $\left(f_{\text {ref }}\right)$, the velocity of the buoy $(\dot{x})$ and the flow rate of the motor $\left(q_{\mathrm{m}}\right)$ as inputs, and provides the corresponding PTO

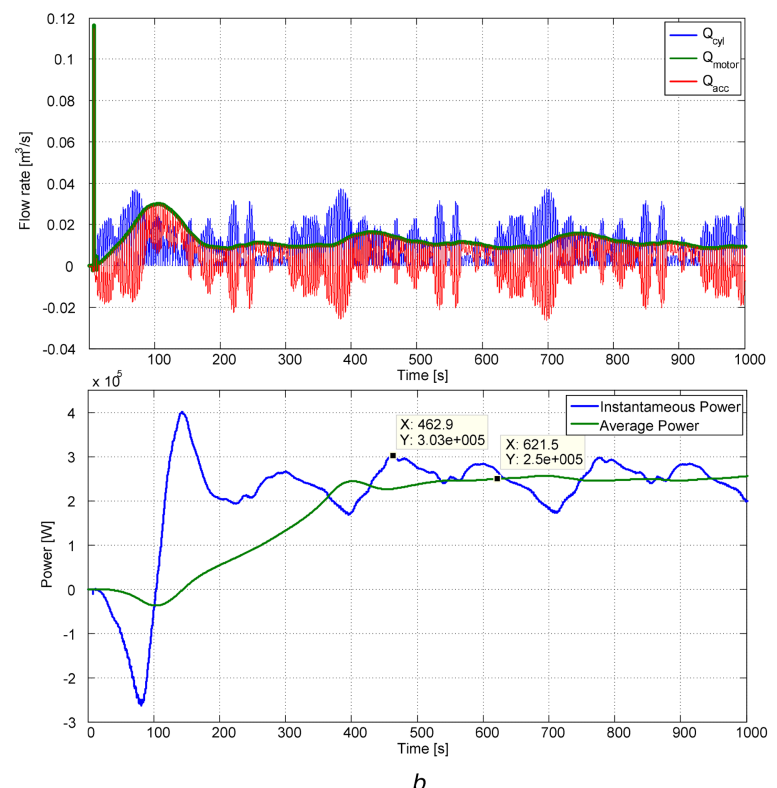

Fig. 4

(a) Overall WEC control strategy emphasising the control system implemented in the PTO, (b) Hydraulic PTO flow rates over the rectified cylinder flow, hydraulic motor and high pressure accumulator, and instantaneous and average power absorption from the hydraulic motor 


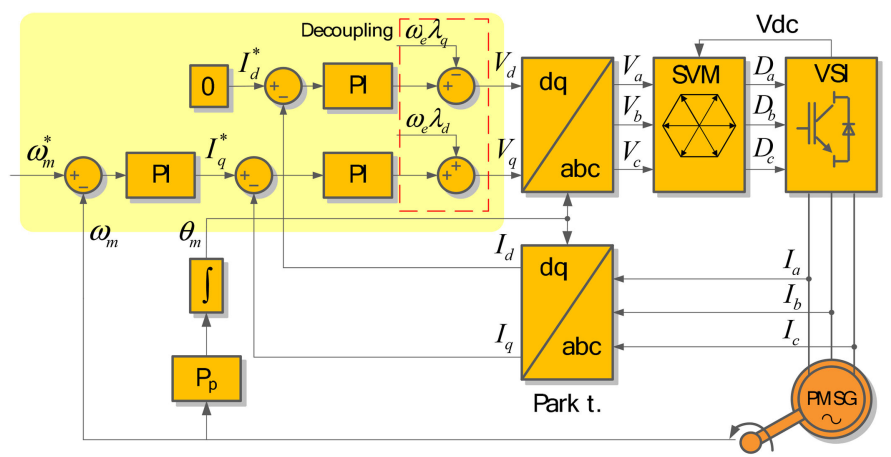

$a$

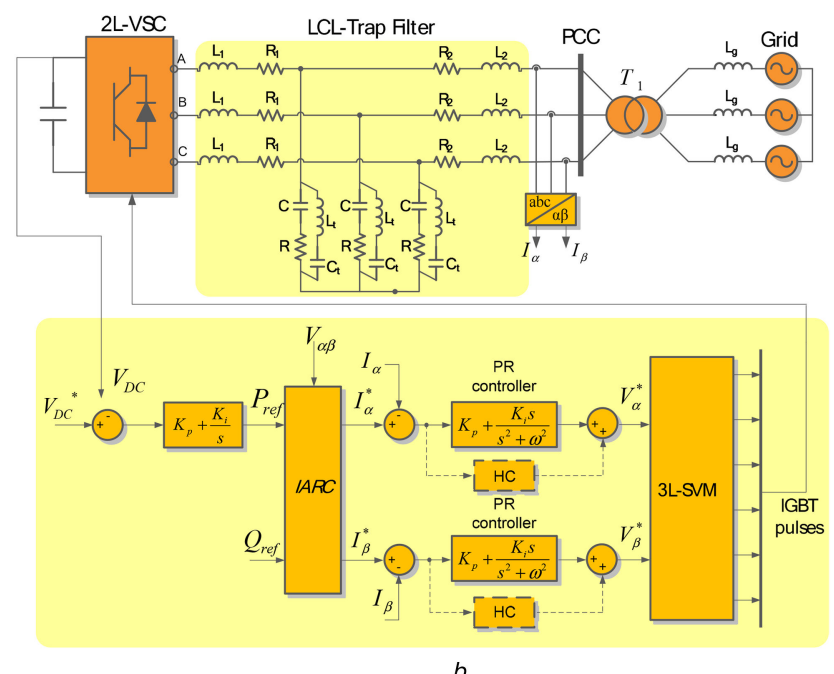

Fig. 5

(a) Block diagram of the field oriented vector control applied in the PMSG, (b) Overall grid connected converter control system

force output $\left(f_{\text {PTO }}\right)$. The main key factors in achieving a suitable control solution rely on a proper sizing of the accumulator capacity $(C)$ and a proper tuning of the proportional-integral (PI) controller, which should have a bandwidth in concordance with the capacitor size. The reference of the hydraulic motor pressure $\left(p_{\text {ref }}\right)$ is calculated according to the expression from

$$
p_{\text {ref }}=\frac{(1 / T) \int_{0}^{T}\left(f_{\text {ref }} \cdot \dot{x}\right)}{q_{\mathrm{m}}}
$$

The hydraulic accumulator capacity as well as the PI controller appear as a result of a desired peak-to-average ratio. In this particular case, the system has been designed for achieving a peakto-average ratio of 1.2 at the output of the hydraulic system. Therefore, the plant and the PI controller parameters are introduced in (19) and (20), respectively,

$$
\begin{gathered}
G_{\text {plant }}=\frac{1}{C s}=\frac{1}{0.0033 \times 10^{-6} \mathrm{~s}} \\
\underset{\text { tustin }}{\Rightarrow} G_{\text {plant }}(z)=\frac{4.5 \times 10^{6} z+4.5 \times 10^{6}}{z-1} \\
C_{\mathrm{PI}}=\frac{K_{\mathrm{p}} z+\left(K_{\mathrm{i}}-K_{\mathrm{p}}\right)}{z-1}=\frac{7.522 \times 10^{-12} z-7.494 \times 10^{-12}}{z-1}
\end{gathered}
$$

The flow rates over the entire system (cylinder, motor and accumulator), and the instantaneous and average power absorption is depicted in Fig. $4 b$. These figures demonstrate the suitable performance of the designed hydraulic PTO system, where the accumulator appears in change of smoothing the instantaneous power fluctuations provided by the cylinder, while keeping the peak-to-average ratio specified in the design requirements.

\section{Modelling and control of PMSG and grid connected power electronic converters}

The implemented PMSG and grid connected control structures can be observed in Figs. $5 a$ and $b$, respectively. For the PSMG, the synchronous reference frame field oriented control has been implemented, as it constitutes one of the most well proven and robust modelling and control approach for achieving variable speed operation of generators [25-27]. In the case of the front-end grid connected converter, a stationary reference frame vector control has been considered for the currents injection to the grid [28,29].

The PMSG control system structure from Fig. $5 a$ is characterised by the implementation of two cascaded control loops, the internal loop (Fig. $6 a$ ) has been tuned according to the optimum modulus criterium [30], which is based on cancelling the pole of the plant with the zero of the controller. In this case, the plant poles were fixed, while the zero of the controller was introduced as the design parameter. Since the WEC performance responds to slow motion dynamics, there was not any strict stabilisation time requirement for the current controller loop. The resulting PI tuning parameters are given as

$$
\begin{aligned}
C_{\text {PI - current }} & =\frac{K_{\mathrm{p}} z+\left(K_{\mathrm{i}}-K_{\mathrm{p}}\right)}{z-1} \\
& =\frac{0.0398 z-0.03973}{z-1} \\
& \Rightarrow K_{\mathrm{p}}=0.0398, K_{\mathrm{i}}=7 \times 10^{-5}
\end{aligned}
$$

For the speed loop, the design criterion was to set the stabilisation time of the speed controller around ten times slower than the inner current loop. This feature can be observed in Fig. $6 d$, where the controller tuning parameters appear in (22). A damping factor of 0.7 was also specified as a design requirement.

Finally, it is worth noting that the coupling between the hydraulic and mechanical models is performed by considering the speed of the hydraulic motor as the reference speed for the generator, while the motor torque represents the load torque exerted on the PMSG. The speed control structure (Fig. 6c), has been tuned without considering the inner current control loop due to the decoupled dynamics of the controllers.

$$
\begin{aligned}
C_{\text {PI }- \text { speed }} & =\frac{K_{\mathrm{p}} z+\left(K_{\mathrm{i}}-K_{\mathrm{p}}\right)}{z-1} \\
& =\frac{5.375 z-5.374}{z-1} \\
& \Rightarrow K_{\mathrm{p}}=5.375, K_{\mathrm{i}}=0.001
\end{aligned}
$$

As it was previously outlined in Fig. 1, four $250 \mathrm{~kW}$ wave energy conversion systems are connected together and grid interfaced through a $1 \mathrm{MW}$ front end inverter. A novel grid filter topology was introduced resulting in a reduced overall filter cost and size when compared with the conventional LCL filter [31, 32]. The current and DC voltage controls of the grid connected front-end inverter have been described in detail in [33], and the same tuning parameters apply for the considered controllers. The current controller has been tuned according to the pole-placement method with the goal of achieving a damping factor of 0.7 and settling time within $20 \mathrm{~ms}$ as design specifications. The outer DC-link controller has been also tuned according to the pole-placement method, ensuring a stabilisation time and damping factor or $t_{\mathrm{s}}=0.2$ and $\xi=0.7$, respectively. The tuning parameters of both controllers are given as 

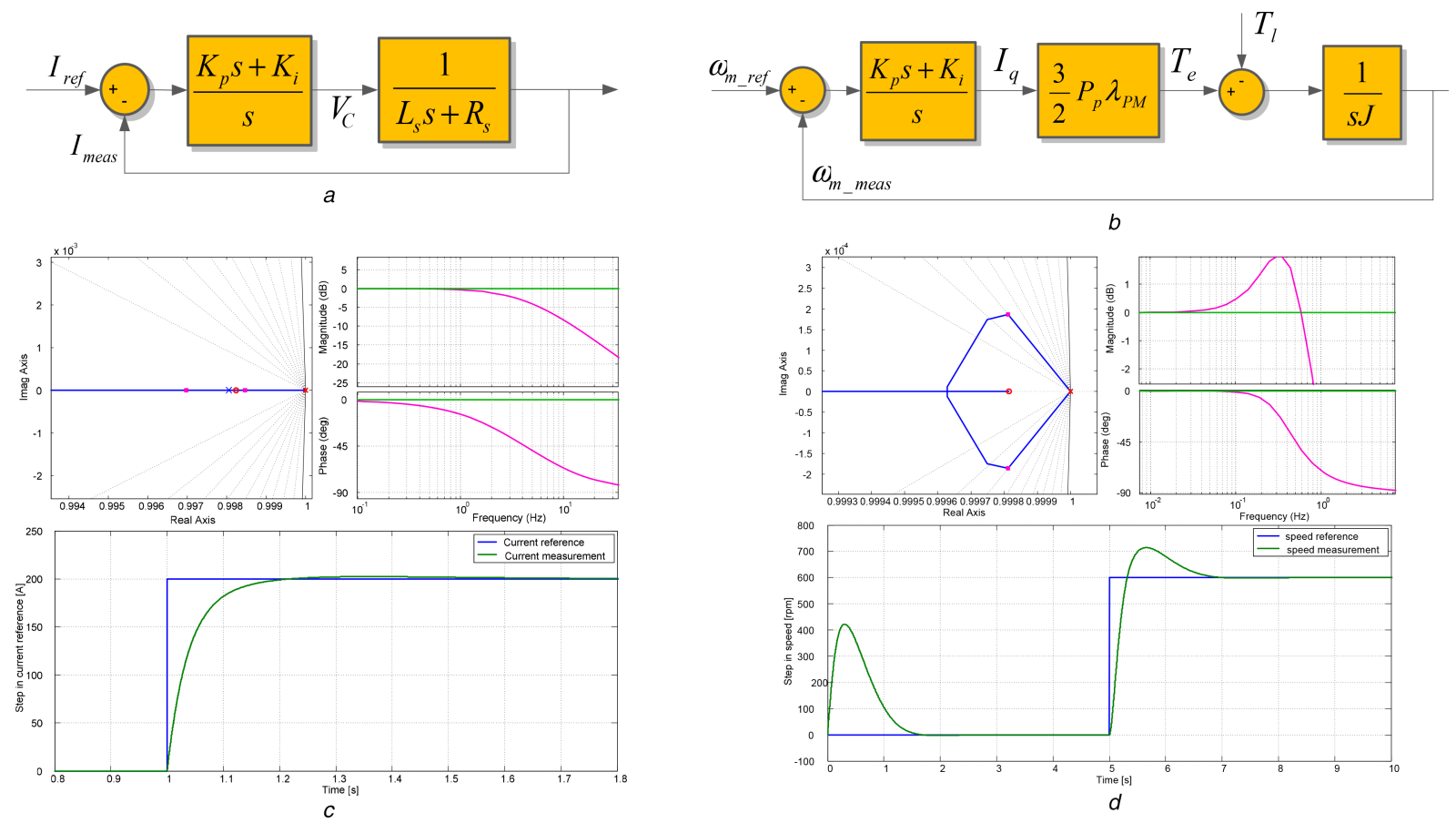

Fig. 6

(a) Current controller loop, (b) Root locus diagram, Bode plot and step response of the closed-loop current controller, (c) Speed controller loop, (d) Root locus diagram, Bode plot and step response of the closed-loop speed controller
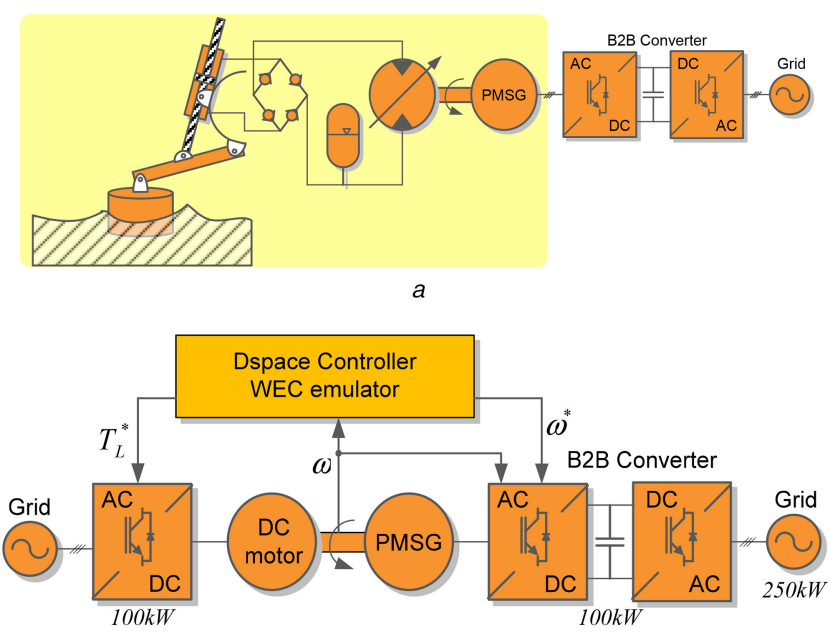

\section{Simulation and experimental results of the proposed WEC controller performance}

The proposed adaptive vector controller has been implemented both in the laboratory and in simulations in order to validate its power extraction capabilities. Due to the lack of available resources, the individual performance of a single WEC has been validated in the laboratory, while the overall wave-to-wire model proposed has been implemented only in simulation.

\subsection{Experimental results of a single wave WEC performance}

The experimental setup is based on a $100 \mathrm{~kW}$ WEC test bench, as the one introduced in Fig. 7a. Such setup comprises a single WEC, of the four paralleled ones proposed in the wave-to-wire model from Fig. 1. As it can be observed in Fig. $7 b$, the hydrodynamic and hydraulic WEC models have been implemented along with the proposed adaptive vector controller in a real-time hardware-in-loop system based on a dSPACE ds 1103 real-time controller, which is interfaced with a PMSG generation test bench. The PMSG test bench is based on a DC motor, emulating the hydraulic motor force acting on the shaft, and a PMSG. Therefore, by measuring the shaft speed, the dSPACE controller is able to determine the torque and speed references that should be, respectively, provided to the motor driver and the PMSG controller for achieving maximum power extraction from the waves. The laboratory setup is introduced in Fig. $7 c$.

The detailed dSPACE and test bench parameters can be

(a) Single WEC laboratory test case, (b) Experimental WEC setup

Table 1 Detailed dSPACE and rated test bench parameters

\begin{tabular}{lc}
\hline dSPACE controller & Laboratory setup \\
\hline$T_{\mathrm{s}}=0.1 \mathrm{~s}$ & $V_{\mathrm{PMSM}}=400 \mathrm{~V}$ \\
$T_{1}=7.7 \mathrm{~s}$ & $\omega_{\mathrm{PMSG}}=1500 \mathrm{rpm}$ \\
$H_{1 / 3}=1.47 \mathrm{~m}$ & $T_{\mathrm{PMSG}}=553 \mathrm{rpm}$ \\
$K_{\mathrm{SOGI}}=\sqrt{2}$ & $V_{D C_{-} B 2 B}=800 \mathrm{~V}$ \\
$Y=0.16$ & $V_{A C_{-} \text {grid }}=400 \mathrm{~V}$ \\
\hline
\end{tabular}

$$
\begin{aligned}
& K_{p \_ \text {current }}=0.353184, K_{i \_ \text {current }}=0.345335 \\
& \quad \text { and } K_{P \_D C \_v o l t a g e}=460, K_{I \_D C \_v o l t a g e}=105799.9
\end{aligned}
$$
laboratory prototype. The converter details used in the present work follow the typical two-level H-bridge back-to-back VSI topology designs. Such electrical power conversion technology has been selected as they already proved their capabilities in the wind energy sector, ensuring a robust, reliable and well known solution in grid connected variable speed generators.

From the hydrodynamic results obtained in Fig. $8 a$, it can be observed that the proposed adaptive vector controller achieves maximum average power absorption from the resource, as the absorbed average power from the PTO $\left(P_{\text {abs }}\right)$ equals the average radiation power $\left(P_{\mathrm{rad}}\right)$, being at the same time half of the average resource power $\left(P_{\text {exc }}\right)$. This system behaviour was introduced in [21] where the optimum operation conditions were described. According to these, the maximum average power absorption of the 

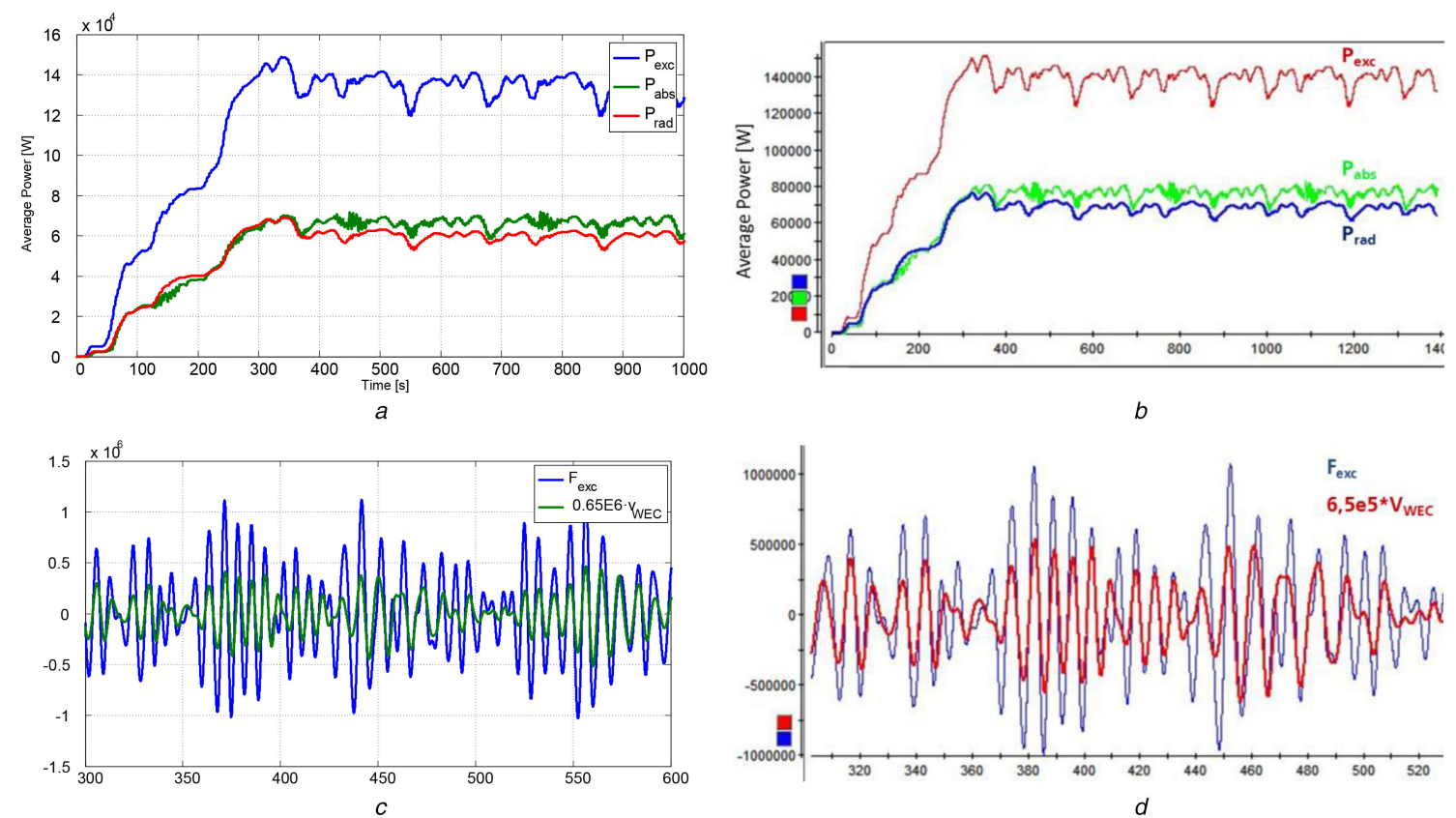

Fig. 8

(a) Comparison of average absorption, excitation and radiation powers when the average power-based vector controller is applied in simulation, (b) Comparison of average absorption, excitation and radiation powers when the average power-based vector controller is applied in laboratory, (c) Comparison of WEC velocity and excitation force in simulation, (d) Comparison of WEC velocity and excitation force in laboratory
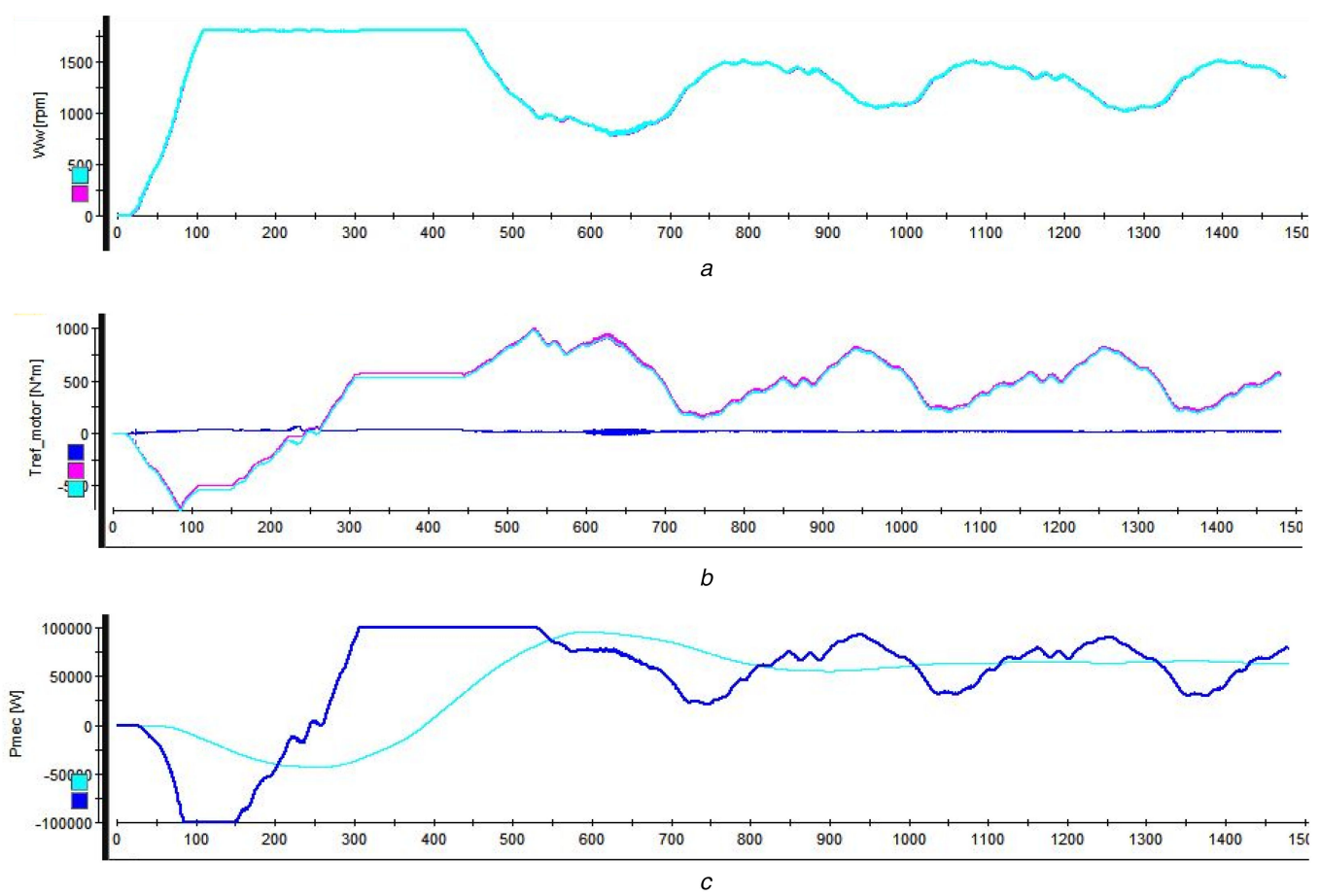

Fig. 9

(a) PMSG generator speed reference (magenta) and measurement (cyan), (b) DC motor torque reference (magenta) and measurement (cyan), (c) Instantaneous (blue) and average (cyan) mechanical power

resource is limited to its radiation losses. In addition, Fig. $8 b$ verifies that the proposed controller instantaneously operates at resonant conditions, as the buoy velocity should appear in phase with the excitation force. In this way, the intrinsic WEC reactive terms do not add any phase shift, since they are cancelled by the virtual buoyancy term introduced from the PTO. As a result, these conditions lead to maximum net power transfer from the wave energy resource to the PTO.

As it can be observed from Fig. 8 the experimental results obtained are quite similar to the simulation ones, thus a suitable validation of the proposed adaptive vector controller has been achieved, for maximum power extraction under irregular sea states.
Finally, the instantaneous behaviour of the mechanical test bench is presented in Fig. 9. From Figs. $9 a$ and $b$, it can be observed that the PMSG speed control and DC motor torque control provide a suitable system performance, as in both cases the measured variables instantaneously follow the references provided from the proposed controller. It is also worth noting that some limitations have been added to the speed and torque references with the purpose of keeping the experimental setup under safe operating conditions. As a last step, the instantaneous and average mechanical power extracted is introduced in Fig. $9 b$, from where it can be also observed that the system performs under the desired setup ratings. 

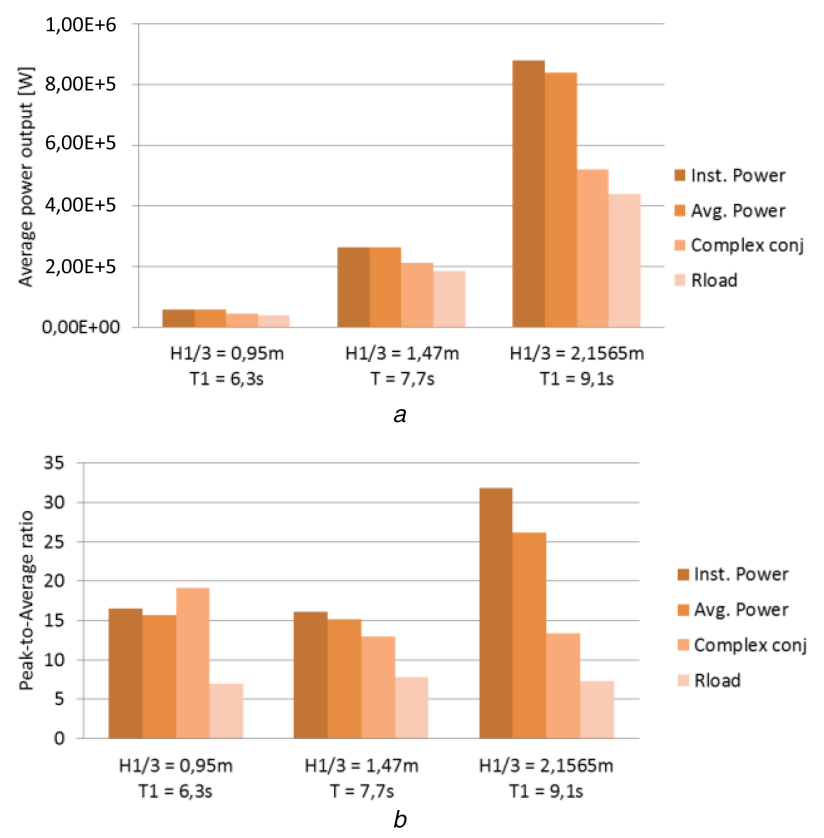

Fig. 10

(a) Average power control strategies comparison when considering different sea state conditions, (b) Peak-to-average control strategies comparison when considering different sea state conditions
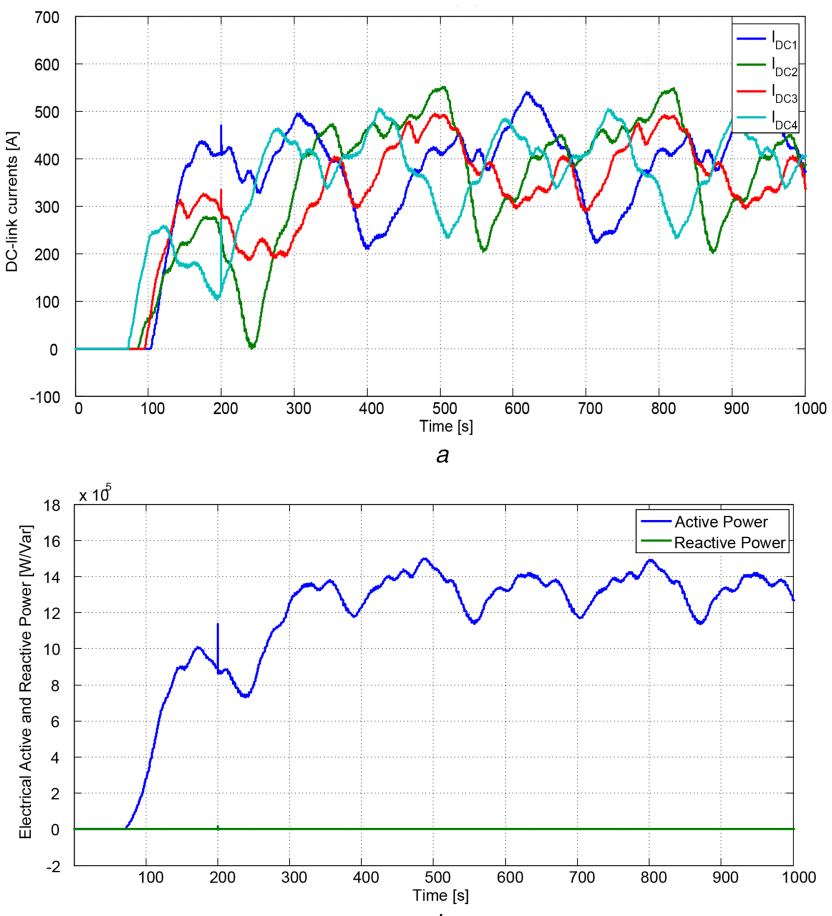

$b$

Fig. 11

(a) DC-link current contribution from each of the PMSGs, (b) Instantaneous active and reactive powerS supply to the grid

From the experimental setup implementation, it is worth noting that no major issues were found in terms of computational burden for achieving a suitable real-time performance. This is due to the fact that the proposed controller is based on a real-time linear control strategy, avoiding any optimisation related process which could take more computational effort, and finally introduce additional delays. In addition, the wave energy field has considerably slow dynamics (in the range of hundreds of milliseconds), so the proposed controller could be implemented in generic DSP processors as the ones found in grid connected converter applications.
6.2 Simulation results of the entire four-WECs wave-to-wire model

The entire wave-to-wire energy conversion system proposed in Fig. 1 has been implemented in Matlab Simulink in order to observe the real-time performance of the proposed adaptive vector controller, as well as its grid integration. As earlier described, the wave energy converter under test is built-up around four wave energy devices of $250 \mathrm{~kW}$ each. The wave energy is extracted from the resource through a hydraulic PTO directly coupled to a PMSG. The electrical power is processed by a common DC-link interconnection, which collects the aggregated instantaneous power from each of the PMSGs and feeds it to a single $1 \mathrm{MW}$ front end VSI.

In order to have a meaningful evaluation of the real benefits introduced by the proposed wave energy control strategy, the performance of the instantaneous and average power theory controllers from (13) and (14) to (15) and (14), respectively are compared with the conventional complex conjugate and passive loading control solutions [10, 15]. From Fig. $10 a$, it can be observed that the proposed instantaneous and average power controllers considerably increase the average output power extraction capabilities of the wave energy converter under a set of different wave energy conditions. This average output power increase comes due to the capability of the proposed controller to adaptively resonate with the most energetic frequency components of the incoming wave energy resource. Opposing to the reactive control methods, where resonance only occurs at the dominant sea state level, the proposed adaptive vector controller is able to ensure instantaneous resonance conditions, leading to maximum energy extraction at the wave-to-wave level. In addition, it can be observed that enhanced power extraction capabilities can be achieved for the proposed controllers in the cases where the wave energy resource deviates more from the WEC design sea state conditions (most probable wave period $T_{\text {wave }}=7.7 \mathrm{~s}$ ), as the proposed wave energy controller ensures resonance conditions regardless of the dominant wave frequency components.

Regarding the peak-to-average ratios achieved from the controller strategies analysed here, it can be clearly observed from Fig. $10 b$ that the average power based strategy provides reduced peak-to-average rates in comparison with the instantaneous power theory. This reduction in the peak-to-average ratio is achieved thanks to the capability of maximising the average power extraction instead of the instantaneous one. Therefore, it can be concluded that the vector controller structure based on average power extraction appears as the most suitable control strategy among the options analysed, as it ensures maximum average power extraction levels while contributing in the reduction of its peak-toaverage ratio.

Regarding the proposed wave-to-wire energy conversion chain, the system described in Fig. 1 has been introduced in simulation by using Matlab Simulink, so the instantaneous control of the entire system can be achieved. From Figs. $11 a$ and $b$, it can be observed how the DC power aggregation produces a smoothing affect over the final output power supplied to the grid. Fig. 11a presents the instantaneous DC currents evolution supplied by each of the PMSGs to the common DC bus. From Fig. 11b, it can be observed that the average produced power is slightly $>1 \mathrm{MW}$ due to the instantaneous aggregation of the power delivered by each of the buoys. From this figure, it can be observed that the peak-to-average ratio has been reduced from 1.2 in the hydraulic circuit, to 1.13 in the electrical output power. This reduction has been achieved from the aggregation effect of the four wave energy converter buoys interconnected in the common DC circuit due to the nonsimultaneity of the individual PMSGs pulsating powers supplied.

Therefore, the aggregation of all different WEC power supplies allows for a reduction of the final peak-to-average ratio obtained in the electrical point of interconnection, with no need of additional electrical storage solutions that would result in dedicated specific converter designs and related costs. In this manner, it is possible to achieve the desired WEC grid integration performance by making use of conventional two-level VSIs, typically implemented in grid connected applications. 


\section{Conclusions}

This paper presents an entire wave-to-wire system configuration and control, which focus on maximum power absorption while reducing as much as possible the electrical power oscillations through the entire energy conversion chain. In addition, this paper proposes a novel wave energy converter control technique based on an adaptive vector controller for maximum power absorption of the wave energy resource. This controller provides a differential contribution in the field of control of wave energy converters, since it provides an instantaneous adaptive behaviour thanks to the implementation of a FLL signal monitoring and synchronisation systems, being able to achieve maximum energy absorption from the resource at any time, regardless of the dominant frequency characteristics of the resource. In addition, it arises as a robust solution, since the controller feedback is based on the instantaneous velocity of the device itself, and not on estimations from far reached measurement buoys.

The entire wave-to-wire energy conversion chain is also found as a novel contribution, as it tries to minimise all possible electrical power oscillations, while the primary resource controller maximises the average power extraction. Thanks to the smoothing effect from DC interconnection of several WECs, the proposed system contributes in reducing further the output power oscillations supplied to the grid. Therefore, the proposed energy conversion chain provides a considerable instantaneous power oscillation reduction due to the inherent storage capability of the PTO and thanks to the electrical interconnection system, which benefits from the aggregation effect of multiple buoys. The proposed electrical interconnection system appears as a suitable solution as well, in case electrical energy storage system would be required, as it could be directly interconnected in the DC link, where the contribution from each of the wave energy converters sum up to reach the final output power.

\section{References}

[1] Margheritini, L., Hansen, A.M. et al.: 'A method for EIA scoping of wave energy converters - based on classification of the used technology', Environ. Impact Assess. Rev., 2012, 32, (1), pp. 33-44

[2] Clément, A., McCullen, P., et al.: 'Wave energy in Europe: current status and perspectives', Renew. Sustain. Energy Rev., 2002, 6, (5), pp. 405-431

[3] Khan, J., Bhuyan, G.S., Moshref, A et al: 'An Assessment of variable characteristics of the pacific northwest region's wave and tidal current power resources, and their interaction with electricity demand and implications for large scale development scenarios for the region' (Powertech Labs Inc., 2008)

[4] Khan, J., Bhuyan, G.S., Moshref, A.: 'Potential opportunities and differences associated with integration of ocean wave and marine current energy plants in comparison to wind energy', a report prepared by Powertech Labs for the IEA-OES, 2009

[5] Khan, J., Bhuyan, G.S. et al: 'Ocean wave and tidal current conversion technologies and their interaction with electrical networks'. Power and Energy Society General Meeting - Conversion and Delivery of Electrical Energy in the 21st Century, 2008, 2008

[6] Falcão, A.F.d.O.: 'Wave energy utilization: A review of the technologies', Renew. Sustain. Energy Rev., 2010, 14, (3), pp. 899-918

[7] Drew, B., Plummer, A.R., et al.: 'A review of wave energy converter technology', Proc. Inst. Mech. Eng. A, J. Power Energy, 2009, 223, (8), pp. 887-902

[8] Czech, B., Bauer, P.: 'Wave energy converter concepts: design challenges and classification', IEEE Ind. Electron. Mag., 2012, 6, (2), pp. 4-16

[9] O'Sullivan, D.L., Dalton, G., et al.: 'Regulatory, technical and financial challenges in the grid connection of wave energy devices', IET Renew. Power Gener., 2010, 4, (6), pp. 555-567
[10] Hals, J., Falnes, J., Moan, T.: 'A comparison of selected strategies for adaptive control of wave energy converters', J. Offshore Mech. Arct. Eng., 2011

[11] Nielsen, S.R.K., Zhou, Q., Kramer, M.M., et al.: 'Optimal control of nonlinear wave energy point converters', Ocean Eng., 2013, 72, pp. 176-187

[12] Hals, J., Falnes, J., Moan, T.: 'Constrained optimal control of a heaving buoy wave energy converter', J. Offshore Mech. Arct. Eng., 2011, 133

[13] da Costa, J.S., Beirao, P., Valerio, D. 'Internal model control applied to the Archimedes wave swing'. Int. Conf. on Systems and Computer Science, 2007

[14] Fusco, F., Ringwood, J.V.: 'A simple and effective real-time controller for wave energy converters', IEEE Trans. Sustain. Energy, 2013, 4, (1), pp. $21-$ 30

[15] Tedeschi, E., Carraro, M., Molinas, M., et al.: 'Effect of control strategies and power take-off efficiency on the power capture from sea waves', IEEE Trans. Energy Convers., 2011, 26, (4)

[16] Molinas, M., Skjervheim, O., et al.: 'Power electronics as grid interface for actively controlled wave energy converters'. Int. Conf. on Clean Electrical Power, 2007, ICCEP '07, 2007

[17] O'Sullivan, D.L., Dalton, G.: 'Challenges in the grid connection of wave energy devices'. Wave and Tidal Energy, European Conf. in (EWTEC), 2009

[18] Michel, W.H.: 'Sea spectra revisited', Marine Technol., 1999, 36, (4), pp. 211-227

[19] Ricci, P., Saulnier, J.B., de O. Falcao, A.F., et al.: 'Time domain models and wave energy converters performance assessment'. Proc. 27th Int. Conf. on Offshore Mechanics and Artic Engineering, Estoril, Portugal, 15-20 June 2008, pp. 1-10

[20] Taghipour, R., Perez, T., Moan, T.: 'Hybrid frequency-time domain models for dynamic response analysis of marine structures', Ocean Eng., 2008, 35, (7)

[21] Falnes, J.: 'Ocean waves and oscillating systems: linear interaction including wave-energy extraction' (Cambridge University Press, 2004)

[22] Cantarellas, A.M., Remon, D., Koch-Ciobotaru, C., et al.: 'Adaptive power control of wave energy converters for maximum power absorption under irregular sea-state conditions'. 2015 IEEE Energy Conversion Congress and Exposition (ECCE), Montreal, QC, 2015, pp. 6655-6659

[23] Teodorescu, R., Liserre, M., Rodriguez, P.: 'Grid converters for photovoltaic and wind power systems' (Wiley-IEEE Press, 2011)

[24] Rodriguez, P., Luna, A., Muñoz-Aguilar, R.S., et al.: 'A stationary reference frame grid synchronization system for three-phase grid-connected power converters under adverse grid conditions', IEEE Trans. Power Electron., 2012, 27, (1)

[25] Bose, B.K.: 'Modern power electronics and AC drives' (Penitence-Hall, 2002)

[26] Krishnan, R.: 'Electric motor drives - modelling, analysis and control' (Penitence-Hall, 2001)

[27] Singh, M., Khadkikar, V., Chandra, A.: 'Grid synchronisation with harmonics and reactive power compensation capability of a permanent magnet synchronous generator-based variable speed wind energy conversion system', IET Power Electron., 2011, 4, (1), pp. 122-130

[28] Blaabjerg, F., Teodorescu, R., Liserre, M. et al.: 'Overview of control and grid synchronization for distributed power generation systems', IEEE Trans. Ind. Electron., 2006, 53, (5), pp. 1398-1409

[29] Vidal, A., Freijedo, F.D., Yepes, A.G., et al.: 'Transient response evaluation of stationary-frame resonant current controllers for grid-connected applications', IET Power Electron., 2014, 7, (7), pp. 1714-1724

[30] Vítečková, M., Víteček, A.: 'Modulus optimum for digital controllers', Acto Montan. Slovaca, 2003, 8, (4), pp. 214-216

[31] Cantarellas, A.M., Rakhshani, E., Remon, D., et al.: 'Design of the LCL+trap filter for the two-level VSC installed in a large-scale wave power plant'. 2013 IEEE Energy Conversion Congress and Exposition, Denver, CO, 2013, pp. $707-712$

[32] Sanatkar-Chayjani, M., Monfared, M.: 'Design of LCL and LLCL filters for single-phase grid connected converters', IET Power Electron., 2016, 9, (9), pp. $1971-1978$

[33] Cantarellas, A.M., Rakhshani, E., Remon, D., et al.: 'Grid connection control of VSC-based high power converters for wave energy applications'. 39th Annual Conf. of the IEEE Industrial Electronics Society, IECON 2013, 10-13 November 2013, pp. 5092-5097 